Article

\title{
Antibodies for Venezuelan Equine Encephalitis Virus Protect Embryoid Bodies from Chikungunya Virus
}

\author{
Emily M. Schultz ${ }^{1}$, TyAnthony J. Jones, Hannah L. Clamp, Kelli L. Barr ${ }^{1 *}$ \\ 1 Department of Biology, Baylor University Waco, TX, USA; \\ * Correspondence: kelli_barr@baylor.edu; Tel.: +01-254-710-2082
}

\begin{abstract}
Chikungunya virus (CHIKV) is an alphavirus that causes febrile illness punctuated by severe polyarthralgia. After the emergence of CHIKV in the Western Hemisphere, multiple reports of congenital infections were published that documented neurological complications, cardiac defects, respiratory distress, and miscarriage. The Western Hemisphere is endemic to several alphaviruses and whether antigenic cross-reactivity can impact the course of infection has not been explored. Recent advances in biomedical engineering have produced cell co-culture models that replicate the cellular interface at the maternal fetal axis. We employed a trans-well assay to determine if cross-reactive antibodies affected the movement and replication of CHIKV across placental cells and into an embryoid body. The data show that antibodies to Venezuelan equine encephalitis virus (VEEV) significantly reduced CHIKV viral load in embryoid bodies. The data highlight that viral pathogenesis can be cell-specific and that exploiting antigenic cross-reactivity could be an avenue for reducing the impact of congenital CHIKV infections.
\end{abstract}

Keywords: Chikungunya, congenital infection, antibody cross-reactivity

\section{Introduction}

Chikungunya virus (CHIKV) is an alphavirus vectored by Aedes mosquitos. In humans, CHIKV can cause a febrile illness punctuated by severe polyarthralgia. Historically, CHIKV infection was thought to be self-limited but increasing reports are showing that rheumatic and neurological sequelae can linger for years following infection [1-3]. When CHIKV emerged in the Western Hemisphere, neuroinvasive disease and congenital infections were reported at rates much higher than the Eastern Hemisphere [4]. CHIKV congenital infections were first reported during the 2005 outbreak on Reunion Island [5-7]. After the emergence of CHIKV in the Western Hemisphere, multiple reports of congenital infections were published that documented neurological complications, cardiac defects, respiratory distress, and miscarriage [5,8-12]. While reports of congenital infection were published in many CHIKV-endemic locations, the vast majority originated from South and Central America [4].

South and Central America along with the Caribbean are endemic to several New World alphaviruses including Maradiaga virus (MADV), Mayaro virus (MAYV), Eastern Equine Encephalitis (EEEV), Venezuelan equine encephalitis (VEEV), and Western equine encephalitis (WEEV). Human exposure prevalence for these viruses can be as high as $80 \%$ in some regions $[13,14]$. Recent studies have indicated that antigenic cross-reactivity, antibody-mediated enhancement, and antibody cross-neutralization of alphaviruses can have a significant impact on the course of infection [15-18].

The study of congenital infections with these viruses is problematic since most animal models don't reflect human disease. While research has shown that non-human primates and sheep can serve as 
models, these systems are (expensive, labor intensive, small sample size etc.). Recent advances in biomedical engineering have produced co-culture models using human stem cells or primary cells that replicate the cellular interface at the blood brain barrier and the maternal fetal axis in order to evaluate the movement and effects of exogenous substances [19]. In order to determine if alphaviral antibody cross-reactivity could impact CHIKV congenital infections, we employed a trans well assay to determine if cross-reactive antibodies impacted the pathogenesis or replication of CHIKV across placental cells and into an embryoid body.

\section{Materials and Methods}

Cell Culture and Virus Propagation

Human Induced Pluripotent Stem Cells (ATCC ACS-1019) were cultured in mTeSR1 media (StemCell Technologies) on plates coated with vitronectin XF (Stemcell Technologies). Primary Human Umbilical Vein Endothelial Cells; Normal, Human, Pooled (HUVEC) (ATCC PCS-100-013) were cultured in EndoGRO-MV-VEGF media (MilliporeSigma) containing 5\% FBS. Additionally, human placental cells BeWo (ATCC CCL-98) were cultured in Ham's F-12K (Kaighn's) Medium containing 10\% FBS, penicillin/streptomycin, 1X non-essential amino acids, 1X Glutamax, and 1mM HEPES. Lastly, Cercopithecus aethiops kidney cell line Vero E6 (ATCC CRL-1586) were grown in Dulbecco's modified Eagle's medium (DMEM) with 10\% FBS, supplemented with penicillin/streptomycin, 1X non-essential amino acids, 1X Glutamax, and 1mM HEPES. All cell lines were incubated at $37^{\circ} \mathrm{C} / 5 \%$ CO2. CHIKV (181/25) was obtained from BEI Resources (NR-50345) and expanded once in Vero cells. Polyclonal Anti-Venezuelan Equine Encephalitis Virus, TC-83 (Subtype IA/B) Glycoprotein (antiserum, Goat), NR-9404, was obtained through BEI Resources, NIAID, NIH.

\section{Embryoid Body Formation}

Embryoid Bodies (EBs) were cultured in an AggreWell 400 24-well plate seeded with ACS-1019 cells at a density of $2.4 \times 105$ cells per well following manufacturer's directions (StemCell Technologies). EBs were cultured in AggreWell EB formation media for 72h after which EBs were harvested and divided equally between replicates of each treatment.

\section{Monolayer Infection and Imaging}

Monolayers of BeWo and HUVEC cells were infected with 100 infectious units per well. After 48h, samples were fixed with $4 \%$ Paraformaldehyde and blocked with 5\% lamb serum. Cells were stained with CHIKV monoclonal 3E7b and MAP2 (Novus Biologicals). Slides were mounted with ProLong Gold Antifade Reagent with DAPI (Cell Signaling Technology \#8961S) and images obtained using an Olympus Fluoview 3000 confocal microscope. Images were processed using the Olympus Fluoview FV10-ASW 4.1 software package. Image analysis and fluorescence quantification was performed using Olympus cellSens Dimension Desktop version 2.2. Pairwise comparisons of fluorescence were performed between the control and the infected groups via Student's T-test with a Tukey post hoc test.

\section{Transwell Co-Culture}

Corning 12 mm Transwell@-COL Collagen-Coated 3.0 $\mu \mathrm{m}$ Pore PTFE Membrane Insert (Cat \# 3494) were seeded with HUVEC cells on the basolateral side of the insert at a concentration of $1.0 \times 105$ cells per $200 \mu \mathrm{l}$, and with BeWo cells on the apical side of the insert at a density of with $1.5 \times 105$ cells in $500 \mu l$. The HUVEC monolayer on the basolateral side was achieved using methods described by Aengenheister et al. (2018) [20]. Briefly, inserts were inverted into 6 well plates, with $1 \mathrm{~mL}$ of PBS in one well to ensure sufficient humidity. Rubber spacers (approximately $1.5 \mathrm{~mm}$ thick) were placed on 
the corner of the 6 well plate to lift up the lid slightly and prevent direct contact of the lid with the inverted insert. After the basolateral side was seeded with HUVECs and the lid was replaced, there was slight adhesion between the lid and the media. HUVEC seeded inserts were then incubated at $37^{\circ} \mathrm{C} / 5 \% \mathrm{CO} 2$ for 2 hours, and afterwards the inserts were placed back into the 12 well plate containing fresh HUVEC Media. Co-cultures were incubated for 72 hours with the media being changed every 48 hours until a $100 \%$ confluent layer was observed.

Figure 1. Experimental model of the transwell co-culture assay modified from Campagnolo et al 2018. Co-cultures of BeWo, HUVEC and EB were apically infected with either CHIKV or CHIKV+VEEV antibody.

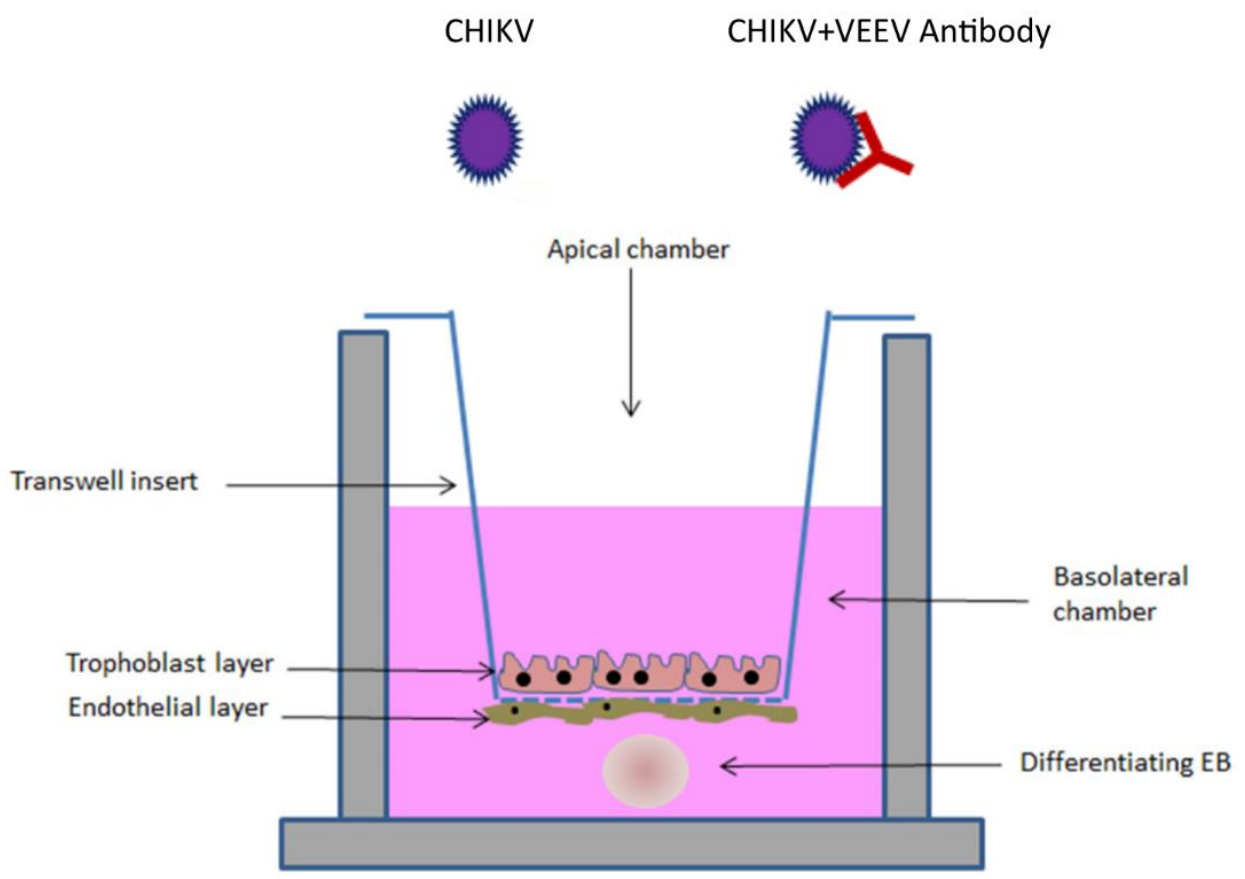

Neutralization Assay

Prior to infection, the media in each basolateral well was replaced with $1 / 2$ HUVEC media 1/2 EB Formation media. EBs were added to the bottom of well. Neutralization assays using VEEV serum were performed using a 1:200 dilution of serum in PBS. 10,000 infectious units of virus in PBS were incubated with serum for 1 hour at $37^{\circ} \mathrm{C}$ after which BeWo cells were inoculated with the mixture. Assay controls included treatments of mock infection and virus only. Culture supernatant (BeWo and HUVEC) and EB samples were taken at 24, 48, and 72 hours. EBs were separated from culture supernatant by centrifugation at 300xg for 4 minutes. The supernatant was aspirated and the EBs were resuspended in PBS and homogenized by vigorous trituration. Results are expressed as an average between 2 independent trials with 3 replicates for each treatment. Percent neutralization was calculated using the virus only treatment as a baseline. Pairwise comparisons were performed between relative treatments using Student's T-test with a Tukey Post-hoc test.

\section{Viral Quantification-}

Plaque assays were performed using culture supernatant from the HUVEC and BeWo monolayers, as well as using the pooled supernatant samples from each treatment at each time point taken during the course of the experiment following method described elsewhere (Barr et al. 2018) [21]. EBs were separated as described above. Briefly, serial dilutions of culture supernatant or EB in PBS were inoculated onto confluent Vero E6 cells and covered with $0.25 \%$ methylcellulose overlay. 
After 3 days, the overlay was removed, and cells were stained with Coomassie blue. Viral RNA was extracted using a kit in accordance to the manufacturer's instructions (Zymo quick Viral RNA kit). For Quantitative Real-Time PCR, Verso One-Step RT-qPCR Kit, SYBR Green, ROX (Thermo Fisher) and primers designed by Patel et al (2019) which are specific for the CHIKV E1 gene were used [22]. Pairwise comparisons between treatments were performed using raw $\mathrm{Ct}$ values with Student's T-test with a Tukey post-hoc test. Relative fold change was calculated via the $\mathrm{Ct}$ method using the non-infected reference cell line as a baseline.

\section{Results}

Chikungunya infects maternal and fetal placental cells

Both BeWo and HUVEC cell lines were infected with CHIKV and visualized using CHIKV monoclonal antibody 3E7b (EMD Millipore). The images show that both BeWo and HUVEC cells are permissive to CHIKV infection with significantly more fluorescence detected in CHIKV-infected cells ( $\mathrm{P}=0.0045,0.012$ respectively) (Figure 2). Neither HUVEC nor BeWo monolayers exhibited noticeable cytopathic effects (CPE) however, there was significantly more immunofluorescence of CHK-MAB with paranuclear staining in BeWo than HUVEC cells (Figure 2) (0.0041). This suggests that BeWo cells may be a more permissive host for CHIKV than HUVEC cells.

Figure 2. CHIKV infection in BeWo and HUVEC cells. 2A. BeWo cells were infected with CHIKV and stained at $48 \mathrm{~h}$ post inoculation. 2B. HUVEC cells were infected with CHIKV and stained at $48 \mathrm{~h}$ post inoculation. (Blue=DAPI, Green=MAP2, Pink= CHIKV) 
A

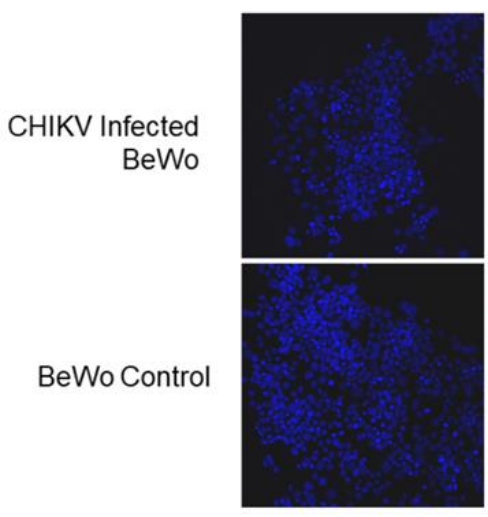

$p=0.452$

B

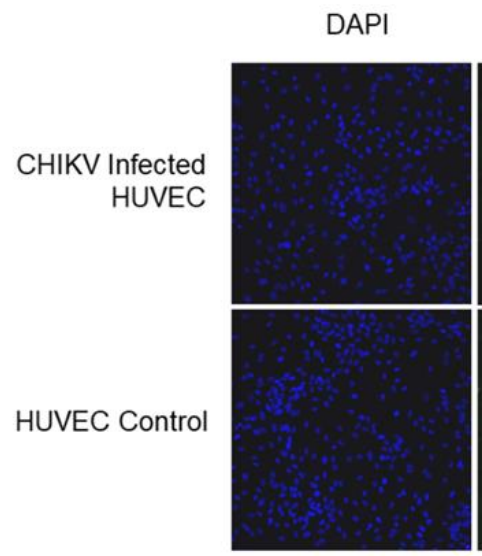

$p=0.081$
MAP2

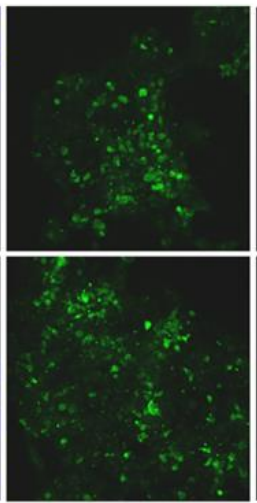

$p=0.583$

MAP2

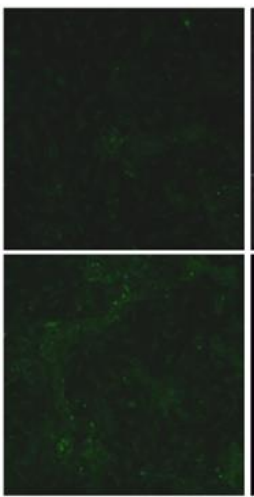

$p=0.082$
CHIKV

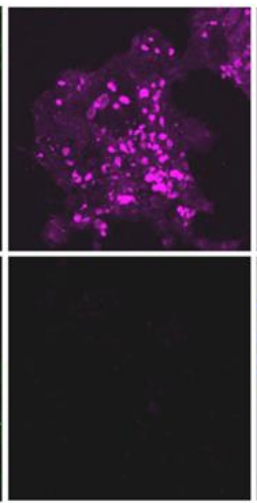

$p=0.004$

CHIKV

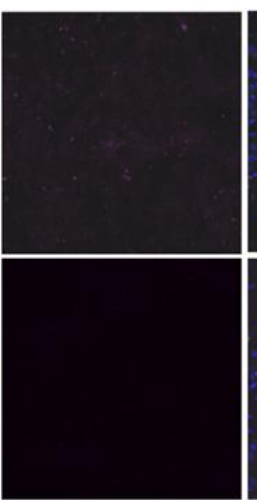

$p=0.012$
MERGED

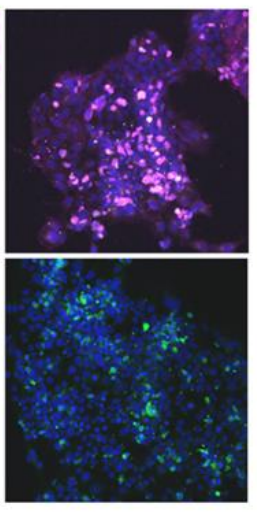

MERGED

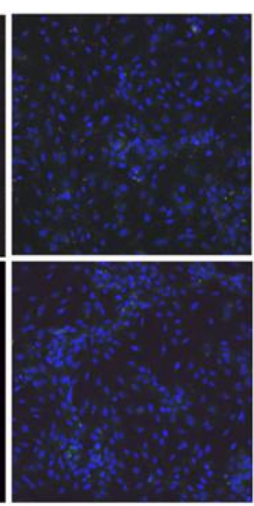

Transwell Assay

CHIKV was detected via viral plaque assay and RT-PCR 24h post infection and continuing through $72 \mathrm{~h}$ post inoculation in BeWo and HUVEC cells. Pairwise comparisons of BeWo cells indicated a significant rise in viral titers at $72 \mathrm{hrs}$ compared with $24 \mathrm{hrs}$ post infection $(\mathrm{P}=0.0387$ ) (Figure $3 \mathrm{~A})$. The presence of VEEV antibodies resulted in a significant reduction in viral plaques at $72 \mathrm{hrs}$ post-inoculation in BeWo cells when compared to CHIKV-infected BeWo cells without VEEV antibodies $(\mathrm{P}=0.0162)$. When evaluated by RT-PCR, significant increases in viral genome copy were observed at all time points for BeWo cells for both CHIKV only $(\mathrm{P}=0.021-0.037)$ and CHIKV + VEEV antibody ( $\mathrm{P}=0.009-0.021)$ treatments (Figure $4 \mathrm{~A}$ ). Pairwise comparisons between CHIKV only and $\mathrm{CHIKV}+$ VEEV antibodies at each time point indicted that no significant changes in $\mathrm{Ct}$ value were found when VEEV antibodies were present in BeWo cells (Figure 4A).

Figure 3. Viral titration of BeWo and HUVEC cell lines. Viral titrations via plaque assay were performed for BeWo and HUVEC cells infected with CHIKV and CHIKV+VEEV antibodies. 3A. Significantly less CHIKV was detected at $72 \mathrm{~h}$ in BeWo cells when VEEV antibodies were present $(\mathrm{P}=0.038)$. 3B. There was no significant difference in $\mathrm{CHIKV}$ at any time point, with or without the presence of VEEV antibodies. 


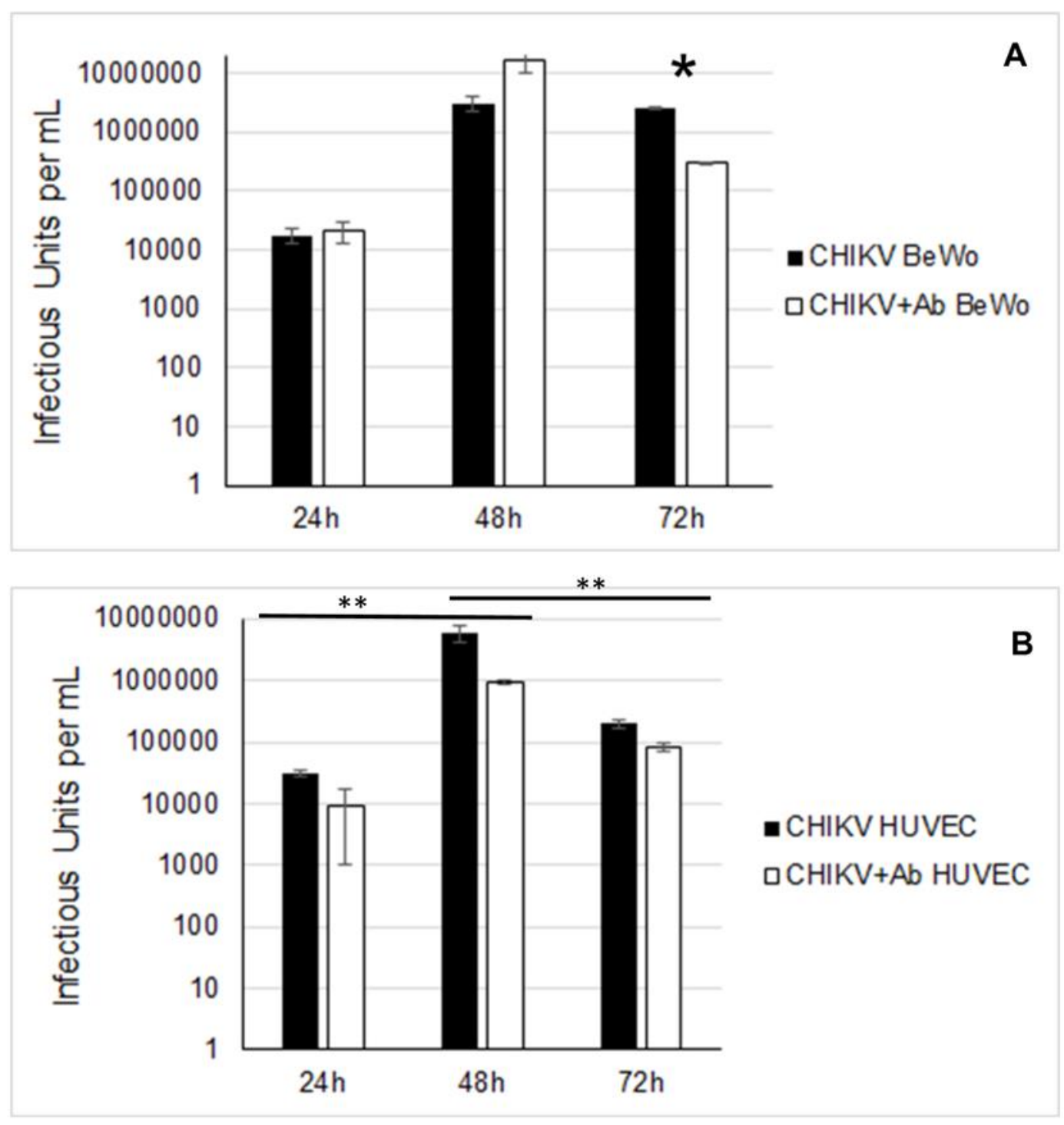

CHIKV was detected in HUVEC cells with significant rises in viral titers at 48 hours compared to $24 \mathrm{hrs}(\mathrm{P}=0.0372)$ followed by significant reduction in viral titers at $72 \mathrm{hrs}$ compared to $48 \mathrm{hrs}(\mathrm{P}=$ 0.0219 ) (Figure 3B). The presence of VEEV antibodies did not result in a significant reduction in viral plaques at any time point in HUVEC cells when compared to CHIKV-infected HUVEC cells without VEEV antibodies. When evaluated by RT-PCR, significant increases in viral genome copy were observed at all time points for HUVEC cells for both CHIKV only ( $\mathrm{P}=0.021-0.037)$ and CHIKV + VEEV antibody $(\mathrm{P}=0.009-0.021)$ treatments (Figure 4B). Pairwise comparisons between CHIKV only and CHIKV + VEEV antibodies at each time point indicted that no significant changes in $\mathrm{Ct}$ value were found when VEEV antibodies were present in HUVEC cells (Figure 4B).

Figure 4. RT-PCR of BeWo and HUVEC cell lines. BeWo cells exhibited no significant differences viral titer when VEEV antibodies were present. 4B. HUVEC cells exhibited no significant differences viral titer when VEEV antibodies were present. 

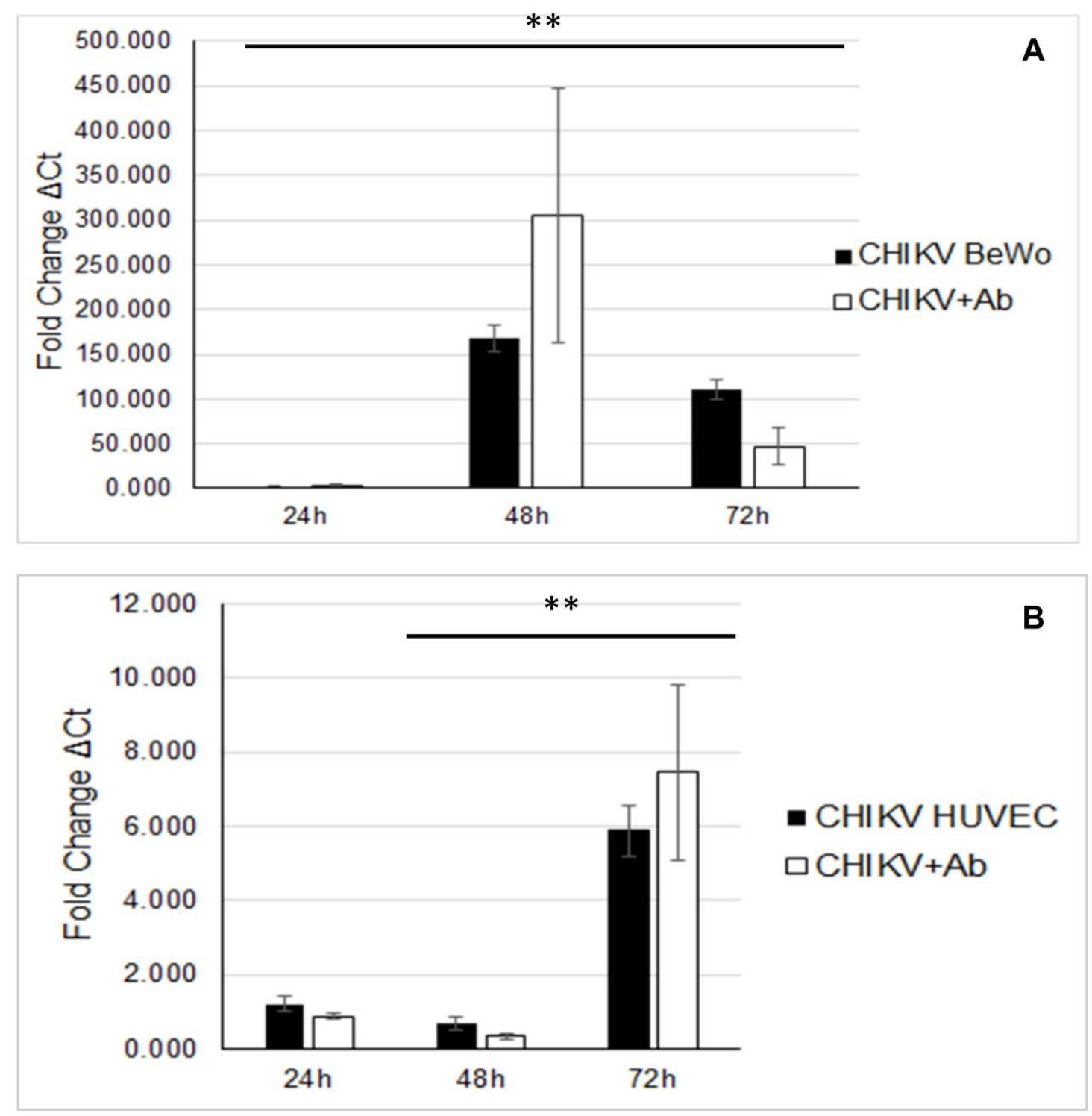

VEEV antibodies reduce CHIKV titers in EB

For EBs, CHIKV was detected by viral plaque assay at 24-, 48- and 72-hours post inoculation (Figure 5). CHIKV was also detected in EBs when VEEV antibody was present at 24-, 48- and 72-hours post inoculation (figure 5). When VEEV antibodies were present, significantly fewer viral plaques were measured at 72 hours post inoculation when compared to the virus only treatment at the same time point ( $\mathrm{P}=0.027$ ) (Figure 5). Conversely, when evaluated by RT-PCR at $72 \mathrm{~h}$ post infection, 5 -fold more viral genome copies were detected for CHIKV alone and 29.95-fold more for CHIKV with VEEV antibodies, which was significantly more than previous time points $(\mathrm{P}=0.002)$. At $72 \mathrm{~h}$ post inoculation, CHIKV was detected at significantly higher levels by RT-PCR when VEEV antibodies were present than $\mathrm{CHIKV}$ alone $(\mathrm{P}=0.0267)$ (Figure 5).

Figure 5. Detection of CHIKV in EBs. 5A. Viral titration of EBs was performed via plaque assay. Significantly less CHIKV was detected at $72 \mathrm{~h}$ post inoculation when VEEV antibodies were present. 5B. RT-PCR detected significantly more genome copies at $72 \mathrm{~h}$ post inoculation when VEEV antibodies were present. 

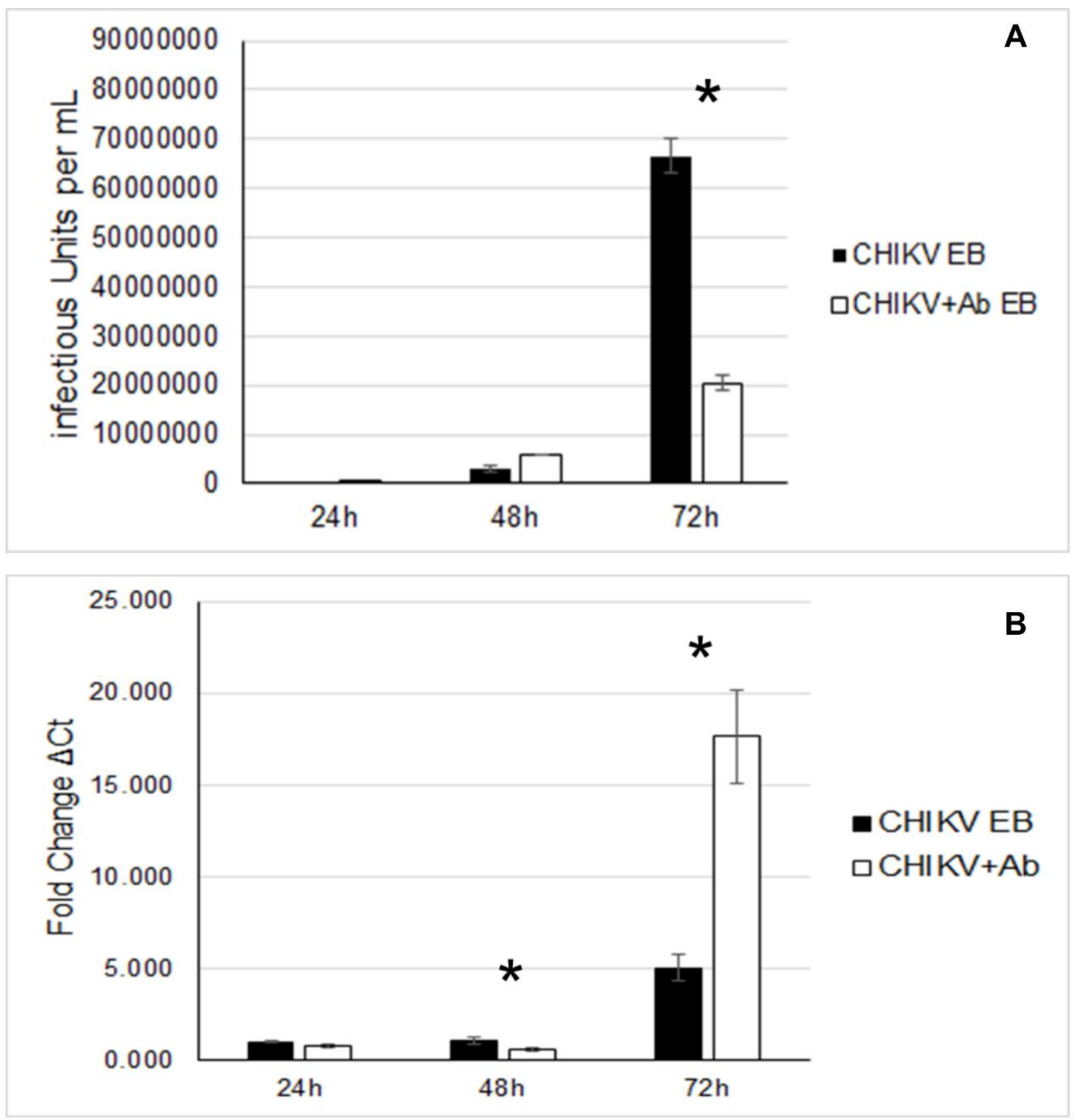

\section{Discussion}

The data show that CHIKV can replicate in maternal and fetal placental cells as well as in EBs. Previous work has shown that the ability for CHIKV to infect and replicate in specific cells types is host specific thus, it is necessary to identify which cells participate in viral pathogenesis [23]. This has also been shown for multiple flaviviruses [24,25]. Reports on CHIKV replication efficiency in mammals and cell lines have shown a range of detection depending on the host [26,27]. The data here show that in BeWo and HUVEC cells, CHIKV replication peaks at 48 hours post inoculation while in EBs, CHIKV replication is greatest at 72 hours post inoculation. Whether this 72 -hour peak reflects an infection delay caused by the movement of CHIKV through 2 cell monolayers and a basement membrane needs further investigation.

CHIKV has been detected in the placenta and amniotic fluid of infected mothers which supports this platform as an in vitro mechanism for studying the kinetics of congenital CHIKV infections $[5,6,28,29]$. Virus was quantified via titration in Vero cells and by RT-PCR. Titration quantifies infectious units while RT-PCR measures genome copies. The data show that there was little correlation between infectious units and genome copies. The data also show that VEEV antibodies 
reduced the number of CHIKV infectious units in EBs by $30 \%$ while increasing genome copies by nearly 2.5 logs. In this study, significantly more copies of the envelope gene were produced than infectious particles by the EBs. This indicates that there could be an issue with viral assembly or that this could be an artifact of the rapidly dividing trilineage cell population of EBs.

BeWo cells are derived from human placenta and were used to represent syncytiotrophoblasts which form the placenta. These cells form a continuous layer around the placenta, are in direct contact with the maternal blood supply, and function in nutrient exchange with the fetus [30]. These cells also form a barrier through which CHIKV must cross in order to invade the fetal environment. HUVEC cells are derived from the fetal umbilical vein and were cultured with a variety of factors to promote microvascular phenotypes. Microvascular placental endothelial cells are involved with placental expansion during the first trimester and vascularization throughout pregnancy [30]. These cells are located adjacent to maternal syncytiotrophoblasts and maternal blood in the intervillous space in the placenta [30,31]. BeWo and HUVEC cells were plated on a permeable membrane that was coated in collagen to provide a basement membrane for cell attachment and for the development of microvillous structures as well as desmosome and tight junctions. The data show that CHIKV actively replicates in both cell lines by 48 hours post inoculation. Whether infection of the basolateral side of the membrane was due to cell: cell contact or virus escape into the basolateral media is not known. However, the detection of CHIKV in cells located on either side of the maternal fetal axis in this study supports case reports of CHIKV congenital infections and isolation of CHIKV from placentas [5,6,28,32].

A major limitation of this study was the omission of Hofbauer cells from the model. These cells function as the antigen presenting macrophages in the placental villous stroma and function in host defense [30]. While the role of Hofbauer cells have not been described for CHIKV, several reports document their role for other congenital viral infections [33-38]. Since Hofbauer cells are antigen presenting, they could play a role in viral enhancement as has been reported for Zika virus [34].

Further, it was found that significantly more genome copies were produced in EBs when VEEV antibodies were present. This might suggest potential antibody mediated enhancement. While this is commonly associated with flaviviruses, antibody mediated enhancement has also been documented for two alphaviruses, Ross River Virus (RRV), and recently, CHIKV (by RT-PCR) [15,39]. These studies measured the enhancement of sub-neutralizing RRV or CHIKV antibodies against subsequent infections with the same virus. However, outside these two studies, antibody mediated cross enhancement has not been explored in depth for other alphaviruses. Regardless, viral plaque assays in this study showed that infection of EBs was significantly reduced when VEEV antibodies were present, suggesting that these antibodies could be interfering with viral assembly, maturation, or exit.

Cross-protective antibodies are commonly targeted for their use in vaccines and therapeutics and antigenic cross-reactivity has been described for CHIKV [40]. This study found that VEEV antibody mediated neutralization of CHIKV occurred in BeWo cells and EBs. The polyclonal serum used in this study was pooled from several goats obtained beginning 2 weeks post final inoculation and continuing for at least 4 weeks. The development of neutralizing antibodies begins about 2 weeks post infection and continues to rise over the next 3-6 months. Thus, the serum used here may not have represented the full repertoire of VEEV neutralizing or enhancing antibodies.

For this study, we used serum from goats vaccinated with TC-83 VEEV vaccine due to its availability from BEI Resources and its defined nature. However, the response of CHIKV to VEEV antibodies may not represent the full potential of CHIKV cross-reactivity in the New World. Although alphaviruses possess a high degree of genetic diversity, phylogenetic studies have shown distinct groupings of Old and New World alphaviruses [41]. The VEEV complex itself contains a high degree of genetic and antigenic variation and human seroprevalence rates have been reported to range from 
14\%-33\% [41-44]. Other New World alphaviruses such as Western Equine Encephalitis and Eastern Equine Encephalitis groups have less genetic diversity than VEEV and are distributed over larger geographic areas than viruses in the VEEV complex [41]. Further, MAYV and MADV are emerging as new threats to human health. MAYV belongs to the Semliki Forest Virus complex which also contains CHIKV and could possess more antigenic cross-reactivity than VEEV [41]. Clearly this area warrants further investigation.

\section{Conclusions}

Early immune VEEV antibodies significantly reduce CHIKV in BeWo and EBs $72 \mathrm{hrs}$ post inoculation. There may be other cross-neutralizing antibodies from other alphaviruses that also impact congenital CHIKV infections. More work that evaluates other alphaviruses and Hofbauer cells is needed.

Author Contributions: KLB and EMS conceived and designed the experiments. KLB, EMS, TJJ. HLC provided logistical and technical support and assisted with editing the manuscript. KLB and EMS drafted the manuscript and all other authors edited and approved the text. All authors have read and agreed to the published version of the manuscript.

Funding: This research received no external funding.

Acknowledgments: This work was supported through start-up funding from Baylor University to Dr. Barr.

Conflicts of Interest: The authors declare no conflict of interest. The funders had no role in the design of the study; in the collection, analyses, or interpretation of data; in the writing of the manuscript, or in the decision to publish the results.

\section{References}

1. Feldstein, L.R.; Rowhani-Rahbar, A.; Staples, J.E.; Weaver, M.R.; Halloran, M.E.; Ellis, E.M. Persistent Arthralgia Associated with Chikungunya Virus Outbreak, US Virgin Islands, December 2014-February 2016. Emerg Infect Dis 2017, 23, 673-676, doi:10.3201/eid2304.161562.

2. Cerny, T.; Schwarz, M.; Schwarz, U.; Lemant, J.; Gérardin, P.; Keller, E. The Range of Neurological Complications in Chikungunya Fever. Neurocrit Care 2017, 10.1007/s12028-017-0413-8, doi:10.1007/s12028-017-0413-8.

3. van Aalst, M.; Nelen, C.M.; Goorhuis, A.; Stijnis, C.; Grobusch, M.P. Long-term sequelae of chikungunya virus disease: A systematic review. Travel medicine and infectious disease 2017, 15, 8-22, doi:10.1016/j.tmaid.2017.01.004.

4. Barr, K.L.; Vaidhyanathan, V. Chikungunya in Infants and Children: Is Pathogenesis Increasing? Viruses 2019, 11, doi:10.3390/v11030294.

5. Lenglet, Y.; Barau, G.; Robillard, P.Y.; Randrianaivo, H.; Michault, A.; Bouveret, A.; Gérardin, P.; Boumahni, B.; Touret, Y.; Kauffmann, E., et al. Chikungunya infection in pregnancy: Evidence for intrauterine infection in pregnant women and vertical transmission in the parturient. Survey of the Reunion Island outbreak. Journal de Gynecologie Obstetrique et Biologie de la Reproduction 2006, 35, 578-583.

6. Touret, Y.; Randrianaivo, H.; Michault, A.; Schuffenecker, I.; Kauffmann, E.; Lenglet, Y.; Barau, G.; Fourmaintraux, A. Early maternal-fetal transmission of the Chikungunya virus. Presse Medicale 2006, 35, 1656-1658, doi:PM-11-2006-35-11-C1-0755-4982-101019-200608574.

7. Robillard, P.Y.; Boumahni, B.; Gérardin, P.; Michault, A.; Fourmaintraux, A.; Schuffenecker, I.; Carbonnier, M.; Djémili, S.; Choker, G.; Roge-Wolter, M., et al. Vertical maternal fetal transmission of the chikungunya virus: Ten cases among 84 pregnant women. Presse Medicale 2006, 35, 785-788, doi:10.1016/S0755-4982(06)74690-5. 
8. Villamil-Gomez, W.; Alba-Silvera, L.; Menco-Ramos, A.; Gonzalez-Vergara, A.; Molinares-Palacios, T.; Barrios-Corrales, M.; Rodriguez-Morales, A.J. Congenital Chikungunya Virus Infection in Sincelejo, Colombia: A Case Series. Journal of tropical pediatrics 2015, 61, 386-392, doi:10.1093/tropej/fmv051.

9. Evans-Gilbert, T. Chikungunya and Neonatal Immunity: Fatal Vertically Transmitted Chikungunya Infection. Am J Trop Med Hyg 2017, 96, 913-915, doi:10.4269/ajtmh.16-0491.

10. Lyra, P.P.; Campos, G.S.; Bandeira, I.D.; Sardi, S.I.; Costa, L.F.; Santos, F.R.; Ribeiro, C.A.; Jardim, A.M.; Santiago, A.C.; de Oliveira, P.M., et al. Congenital Chikungunya Virus Infection after an Outbreak in Salvador, Bahia, Brazil. AJP Rep 2016, 6, e299-300, doi:10.1055/s-0036-1587323.

11. Gerardin, P.; Barau, G.; Michault, A.; Bintner, M.; Randrianaivo, H.; Choker, G.; Lenglet, Y.; Touret, Y.; Bouveret, A.; Grivard, P., et al. Multidisciplinary prospective study of mother-to-child chikungunya virus infections on the island of La Reunion. PLoS Med 2008, 5, e60, doi:10.1371/journal.pmed.0050060.

12. Cardona-Correa, S.E.; Castano-Jaramillo, L.M.; Quevedo-Velez, A. [Vertical transmission of chikungunya virus infection. Case Report]. Revista chilena de pediatria 2017, 88, 285-288, doi:10.4067/s0370-41062017000200015.

13. Vittor, A.Y.; Armien, B.; Gonzalez, P.; Carrera, J.P.; Dominguez, C.; Valderrama, A.; Glass, G.E.; Beltran, D.; Cisneros, J.; Wang, E., et al. Epidemiology of Emergent Madariaga Encephalitis in a Region with Endemic Venezuelan Equine Encephalitis: Initial Host Studies and Human Cross-Sectional Study in Darien, Panama. PLoS Negl Trop Dis 2016, 10, e0004554, doi:10.1371/journal.pntd.0004554.

14. Cardozo, F.; Konigheim, B.; Albrieu-Llinás, G.; Rivarola, M.E.; Aguilar, J.; Rojas, A.; Quaglia, A.I.; Paez, M.; Guillén, Y.; Diaz, A., et al. Alphaviruses: Serological Evidence of Human Infection in Paraguay (2012-2013). Vector-Borne and Zoonotic Diseases 2018, 18, 266-272, doi:10.1089/vbz.2017.2178.

15. Lum, F.M.; Couderc, T.; Chia, B.S.; Ong, R.Y.; Her, Z.; Chow, A.; Leo, Y.S.; Kam, Y.W.; Renia, L.; Lecuit, M., et al. Antibody-mediated enhancement aggravates chikungunya virus infection and disease severity. Sci Rep 2018, 8, 1860, doi:10.1038/s41598-018-20305-4.

16. Kam, Y.W.; Pok, K.Y.; Eng, K.E.; Tan, L.K.; Kaur, S.; Lee, W.W.L.; Leo, Y.S.; Ng, L.C.; Ng, L.F.P. Sero-Prevalence and Cross-Reactivity of Chikungunya Virus Specific Anti-E2EP3 Antibodies in Arbovirus-Infected Patients. In PLoS Negl Trop Dis, 2015; Vol. 9.

17. Partidos, C.D.; Paykel, J.; Weger, J.; Borland, E.M.; Powers, A.M.; Seymour, R.; Weaver, S.C.; Stinchcomb, D.T.; Osorio, J.E. Cross-protective immunity against o'nyong-nyong virus afforded by a novel recombinant chikungunya vaccine. Vaccine 2012, 30, 4638-4643, doi:10.1016/j.vaccine.2012.04.099.

18. Rico, A.B.; Phillips, A.T.; Schountz, T.; Jarvis, D.L.; Tjalkens, R.B.; Powers, A.M.; Olson, K.E. Venezuelan and western equine encephalitis virus E1 liposome antigen nucleic acid complexes protect mice from lethal challenge with multiple alphaviruses. Virology 2016, 499, 30-39, doi:10.1016/j.virol.2016.08.023.

19. L, C.; V, L.; M, M.; A, M.; A, P. In vitro experimental models to study the efficiency of the placental barrier for environmental toxicants: tumor cell lines versus trophoblast primary cells. Biomedicine \& Prevention 2018, 1, doi:DOI:10.19252/00000009D.

20. Aengenheister, L.; Keevend, K.; Muoth, C.; Schonenberger, R.; Diener, L.; Wick, P.; Buerki-Thurnherr, T. An advanced human in vitro co-culture model for translocation studies across the placental barrier. Sci Rep 2018, 8, 5388, doi:10.1038/s41598-018-23410-6.

21. Barr, K.L.; Khan, E.; Farooqi, J.Q.; Imtiaz, K.; Prakoso, D.; Malik, F.; Lednicky, J.A.; Long, M.T. Evidence of Chikungunya Virus Disease in Pakistan Since 2015 With Patients Demonstrating Involvement of the Central Nervous System. Front Public Health 2018, 6, 186, doi:10.3389/fpubh.2018.00186. 
22. B, S.R.; Patel, A.K.; Kabra, S.K.; Lodha, R.; Ratageri, V.H.; Ray, P. Virus load and clinical features during the acute phase of Chikungunya infection in children. PLoS One 2019, 14, e0211036, doi:10.1371/journal.pone.0211036.

23. Wikan, N.; Sakoonwatanyoo, P.; Ubol, S.; Yoksan, S.; Smith, D.R. Chikungunya virus infection of cell lines: analysis of the East, Central and South African lineage. PloS one 2012, 7, e31102-e31102, doi:10.1371/journal.pone.0031102.

24. Barr, K.L.; Anderson, B.D.; Prakoso, D.; Long, M.T. Working with Zika and Usutu Viruses In Vitro. PLoS Negl Trop Dis 2016, 10, e0004931, doi:10.1371/journal.pntd.0004931.

25. KL, B.; BD, A.; GL, H.; JA, F.; GC, G.; DA, F. Dengue serotypes 1-4 exhibit unique host specificity in vitro. Virus Adaptation and Treatment 2012, 2012, 8, doi:https://doi.org/10.2147/VAAT.S36856.

26. Guerrero, N.A.S.; Bello, F.J. Comparative assessment of the replication efficiency of dengue, yellow fever, and chikungunya arboviruses in some insect and mammalian cell lines. Rev Soc Bras Med Trop 2019, 52, e20180511, doi:10.1590/0037-8682-0511-2018.

27. Sasayama, M.; Benjathummarak, S.; Kawashita, N.; Rukmanee, P.; Sangmukdanun, S.; Masrinoul, P.; Pitaksajjakul, P.; Puiprom, O.; Wuthisen, P.; Kurosu, T., et al. Chikungunya virus was isolated in Thailand, 2010. Virus genes 2014, 49, 485-489, doi:10.1007/s11262-014-1105-5.

28. Watanaveeradej, V.; Endy, T.P.; Simasathien, S.; Kerdpanich, A.; Polprasert, N.; Aree, C.; Vaughn, D.W.; Nisalak, A. The study transplacental chikungunya virus antibody kinetics, Thailand. Emerg Infect Dis 2006, 12, 1770-1772, doi:10.3201/eid1211.051560.

29. Prata-Barbosa, A.; Cleto-Yamane, T.L.; Robaina, J.R.; Guastavino, A.B.; de Magalhaes-Barbosa, M.C.; Brindeiro, R.M.; Medronho, R.A.; da Cunha, A. Co-infection with Zika and Chikungunya viruses associated with fetal death-A case report. Int J Infect Dis 2018, 72, 25-27, doi:10.1016/j.ijid.2018.04.4320.

30. Y, W.; S, Z. Chapter 4, Cell Types of the Placenta. In Vascular Biology of the Placenta, Morgan \& Claypool Life Sciences: San Rafael, 2010.

31. K, B.; P, K.; R., B. Anatomy and pathology of the umbilical cord. In Pathology of the human placenta, 5 ed.; K, B., P, K., R., B., Eds. 2006; p. 71.

32. Chen, C.I.; Clark, D.C.; Pesavento, P.; Lerche, N.W.; Luciw, P.A.; Reisen, W.K.; Brault, A.C. Comparative pathogenesis of epidemic and enzootic Chikungunya viruses in a pregnant Rhesus macaque model. Am J Trop Med Hyg 2010, 83, 1249-1258, doi:10.4269/ajtmh.2010.10-0290.

33. Zulu, M.Z.; Martinez, F.O.; Gordon, S.; Gray, C.M. The Elusive Role of Placental Macrophages: The Hofbauer Cell. Journal of innate immunity 2019, 11, 447-456, doi:10.1159/000497416.

34. Zimmerman, M.G.; Quicke, K.M.; O'Neal, J.T.; Arora, N.; Machiah, D.; Priyamvada, L.; Kauffman, R.C.; Register, E.; Adekunle, O.; Swieboda, D., et al. Cross-Reactive Dengue Virus Antibodies Augment Zika Virus Infection of Human Placental Macrophages. Cell Host Microbe 2018, 24, 731-742.e736, doi:10.1016/j.chom.2018.10.008.

35. Reyes, L.; Golos, T.G. Hofbauer Cells: Their Role in Healthy and Complicated Pregnancy. Front Immunol 2018, 9, 2628, doi:10.3389/fimmu.2018.02628.

36. Rosenberg, A.Z.; Yu, W.; Hill, D.A.; Reyes, C.A.; Schwartz, D.A. Placental Pathology of Zika Virus: Viral Infection of the Placenta Induces Villous Stromal Macrophage (Hofbauer Cell) Proliferation and Hyperplasia. Arch Pathol Lab Med 2017, 141, 43-48, doi:10.5858/arpa.2016-0401-OA. 
37. Schwartz, D.A. Viral infection, proliferation, and hyperplasia of Hofbauer cells and absence of inflammation characterize the placental pathology of fetuses with congenital Zika virus infection. Arch Gynecol Obstet 2017, 295, 1361-1368, doi:10.1007/s00404-017-4361-5.

38. Simoni, M.K.; Jurado, K.A.; Abrahams, V.M.; Fikrig, E.; Guller, S. Zika virus infection of Hofbauer cells. Am J Reprod Immunol 2017, 77, doi:10.1111/aji.12613.

39. Linn, M.L.; Aaskov, J.G.; Suhrbier, A. Antibody-dependent enhancement and persistence in macrophages of an arbovirus associated with arthritis. J Gen Virol 1996, 77 ( Pt 3), 407-411, doi:10.1099/0022-1317-77-3-407.

40. Fox, J.M.; Long, F.; Edeling, M.A.; Lin, H.; van Duijl-Richter, M.K.S.; Fong, R.H.; Kahle, K.M.; Smit, J.M.; Jin, J.; Simmons, G., et al. Broadly Neutralizing Alphavirus Antibodies Bind an Epitope on E2 and Inhibit Entry and Egress. Cell 2015, 163, 1095-1107, doi:10.1016/j.cell.2015.10.050.

41. Weaver, S.C.; Winegar, R.; Manger, I.D.; Forrester, N.L. Alphaviruses: population genetics and determinants of emergence. Antiviral research 2012, 94, 242-257, doi:10.1016/j.antiviral.2012.04.002.

42. Carrera, J.P.; Bagamian, K.H.; Travassos da Rosa, A.P.; Wang, E.; Beltran, D.; Gundaker, N.D.; Armien, B.; Arroyo, G.; Sosa, N.; Pascale, J.M., et al. Human and Equine Infection with Alphaviruses and Flaviviruses in Panama during 2010: A Cross-Sectional Study of Household Contacts during an Encephalitis Outbreak. Am J Trop Med Hyg 2018, 98, 1798-1804, doi:10.4269/ajtmh.17-0679.

43. Pisano, M.B.; Oria, G.; Beskow, G.; Aguilar, J.; Konigheim, B.; Cacace, M.L.; Aguirre, L.; Stein, M.; Contigiani, M.S. Venezuelan equine encephalitis viruses (VEEV) in Argentina: serological evidence of human infection. PLoS Negl Trop Dis 2013, 7, e2551, doi:10.1371/journal.pntd.0002551.

44. Adams, A.P.; Navarro-Lopez, R.; Ramirez-Aguilar, F.J.; Lopez-Gonzalez, I.; Leal, G.; Flores-Mayorga, J.M.; Travassos da Rosa, A.P.A.; Saxton-Shaw, K.D.; Singh, A.J.; Borland, E.M., et al. Venezuelan equine encephalitis virus activity in the Gulf Coast region of Mexico, 2003-2010. PLoS neglected tropical diseases 2012, 6, e1875-e1875, doi:10.1371/journal.pntd.0001875. 